\title{
Discussion on Feasibility of Removal of Cognitive Biases
}

\author{
Atsuo Murata \\ Okayama University, Okayama, Japan
}

\begin{abstract}
It is possible that cognitive biases potentially and unexpectedly distort our decision and become a root cause of disasters such as the Three Mile Island disaster and the subprime disaster that vanished pension funds. Using six types of cognitive biases (ignorance of base rate, regression to the mean, conjunction fallacy, framing effect, illusion of covariation, and overconfidence), this study explored whether there are effective countermeasures to remove cognitive biases and prevent our decision from distorting. The debiasing methods of conjunction fallacy and ignorance of base rate were effective to some extent for suppressing the biases. For cognitive biases such as regression to the mean, framing effect, and fallacy of covariation, and overconfidence, the debiasing methods were not necessarily effective for suppressing cognitive biases. Based on the results, some implications for the prevention of undesirable outcomes or events such as disasters, crashes, or collisions were given.
\end{abstract}

Keywords: cognitive biases, debiasing, conjunction fallacy, ignorance of base rate, regression to the mean, framing effect, illusion of covariance, overconfidence, safety management

\section{Introduction}

We generally cannot make decision rationally, and thus suffer from cognitive biases (Kahneman, 2011; Tversky \& Kahneman, 1974, 1984; Altman, 2012; Anger, 2012; Bazerman \& Moore, 2001) due to bounded rationality. Our cognitive information processing is based on either System1, which is simple and intuitive, operates quickly, automatically, without time consuming, and intuitively with little or no efforts, or System2, which requires us to conduct effortful, demanding and deliberate mental activities. When we have no time to deliberate, we tend to adopt heuristic approaches based on System1. However, it is possible that such approaches are frequently got trapped in cognitive biases as pointed out by Murata, Nakamura, and Karwowski (2015).

Bazerman and Moore (2001) hypothesized that availability, representativeness, confirmation, or affect heuristics form the basis of a variety of biases such as availability bias, confirmation bias, hindsight bias, ignorance of base rate, conjunction fallacy, ignorance of sample size, and anchoring and adjustment. They also insisted that not only heuristics but also overconfidence and framing presumably cause a variety of biases. Moreover, they assumed that our bounded awareness and uncertain situations form the basis of heuristics, overconfidence, and framing. Based on such discussion, it seems valid to judge that we sometimes can behave irrationally. In this manner, we at times show a tendency of irrational behavior. In most cases, we tend to be unaware of how irrational behaviors affect us. Such irrational properties unconsciously distort our decisions and, in the worst case, lead to undesirable events or outcomes such as incidents, collisions, disasters, or crashes.

Atsuo Murata, Ph.D., professor, Department of Intelligent Mechanical Systems, Graduate School of Natural Science and Technology, Okayama University. 
As discussed in Murata et al. (2015), our behavior without consideration of our bounded rationality (irrationality) might unexpectedly bring upon us serious disasters, collisions, or crashes such as the Challenger space shuttle disaster (Reason, 1990; Vaughan, 1997), or the Three Mile Island nuclear reactor meltdown (Lusted, 2012; Osif, Baratta, \& Conkling, 2006). Therefore, it is an important issue to investigate whether cognitive biases can be removed somehow.

In Murata, Nakamura, and Kubo (2014), similar cognitive biases were taken up, and it was discussed whether such biases could be removed or not. Although they concluded that the addition of a mean value to the graph or table was effective to the understanding of regression to the mean and removing a cognitive bias related to this phenomenon, the detailed statistical analysis did not support this viewpoint. The conclusions in Murata et al. (2014) were not supported by appropriate statistical analyses. Therefore, it is necessary to reach an appropriate conclusions based on an appropriate analysis of experimental data. Moreover, in Murata et al. (2014), no implications were given on how cognitive biases lead to critical disasters or crashes and how important it is to remove cognitive biases for the prevention of critical and undesirable consequences. In other words, they did not fully state how important it is to develop a debiasing method and attempt to remove cognitive biases as much as one can.

Based on the discussion above, it is important to remove cognitive biases that potentially bring upon undesirable consequences. Gigerenzer (1994) showed that it is possible to remove conjunction fallacy and ignorance of base rate which is regarded to stem from representativeness heuristics to some extent. He verified that the debiasing of these biases was possible by changing the method of information representation from a probability-based to a frequency-based one. Todd and Gigerenzer (2000), Gigerenze, Todd, and ABC Research Group (1999), and Gigerenzer and Goldstein (1996) showed that the simple (fast and frugal) heuristics perform comparably to complex and deliberate algorithms when such fast and frugal heuristics lead to robustness of thinking processes. Haselton et al. (2009) suggested that the research on cognitive biases might be well understood by evolutionary perspective on cognitive bias. Such perspective might be helpful to get insight into the mechanism on how cognitive biases distort our decision and judgment and under what conditions we tend to be vulnerable and suffer from cognitive biases. Larrick (2004) described the sources of biases in decision making. He showed that cognitive biases arise from psychologically-based errors, association-based errors, and strategy-based errors, and classified debiasing strategies into motivational, cognitive, and technological ones. Motivational strategies include incentives and accountability. Cognitive strategies include "consider the opposite", a training in rules, a training in representations, and a training in biases. Technological strategies include a proposal of unbiased decision strategies as a group and the development of a decision support system that can effectively detect cognitive biases. Arkes (1991) also suggested that a few general causes underlie a wide range of cognitive biases, and that recognizing the causes of biases might facilitate predicting when some debiasing strategies would be dominant.

As it is possible that biases in decision making, in the worst case, induce undesirable events or outcomes such as incidents, collisions, crashes, or disasters, the elimination of cognitive biases must be one of the effective and promising countermeasures for preventing undesirable events. Exploring how cognitive biases can be removed quantitatively is important to establish a safety management strategy that does not get trapped in cognitive biases.

This study examined in more detail and systematically (using appropriate statistical analyses) whether cognitive biases such as ignorance of base rate, regression to the mean, conjunction fallacy, framing effect, illusion of covariation, and overconfidence can be eliminated or not. In this manner, the effectiveness of 
debiasing methods of these biases was experimentally discussed, and some implications were given for the importance of further continuing to develop debiasing techniques and attempting to remove cognitive biases that potentially become a risk factor of critical disasters or crashes such as the Three Mile Island meltdown or the Challenger space shuttle disaster.

\section{Method}

\section{Participants}

A total of 80 participants took part in the experiment. The participants were equally allocated to either biasing or debiasing condition. In other words, 40 participants were allocated to either biasing or debiasing condition. The limitation of answering time differed among the six questions below. The participant was required to answer the questions on six types of cognitive biases below in this order. On the basis of the answer for each type of bias, the performance data such as the percentage correct of each question were compared between the biasing and the debiasing condition.

\section{Conjunction Fallacy}

According to Tversky and Kahneman (1983), conjunction fallacy is explained briefly using the probability-based representation below. Although it is rational to choose (a) for the probability-based representation, a lot of people actually tend to choose (b). It is mathematically apparent that the probability of (a) $(\operatorname{Pr}(\mathrm{a}))$ is larger than that of (b), that is, (a) and "Linda is active in the feminist movement" $(\operatorname{Pr}((\mathrm{a})$ and "Linda is active in the feminist movement")). In spite of this, we inevitably mistake that the probability of two events (Linda is a bank teller, and Linda is active in the feminist movement) occurring simultaneously is larger than that of one event (Linda is a bank teller). This bias is called conjunction fallacy.

The participant was presented with the following sentences. Linda is 31 years old, single, outspoken, and active and intelligent. When she was a student, she was enthusiastically concerned herself in issues of discrimination and social justice, and frequently involved with antinuclear demonstrations. The participant was required to answer the question on the basis of own understanding of the sentence above. No time limitation was imposed on the participant. The correct answer corresponds to the alternative (a) above. Choosing the alternative (b) indicates that the participant gets involved in conjunction fallacy. The experimental factor (between-subject factor) was whether debiasing was tried or not. The percentage correct was compared between the biasing and the debiasing condition.

Probability-based (biasing) representation:

Which of the following two alternatives is more probable?

(a) Linda is a bank teller.

(b) Linda is a bank teller. She is also actively engaged in the feminist movement.

Frequency-based (debiasing) representation:

Imagine 200 females like Linda. Which of the following two alternatives is more frequent?

(a) Linda is a bank teller.

(b) Linda is a bank teller. She is also actively engaged in the feminist movement.

\section{Ignorance of Base Rate}

Ignorance of base rate corresponds to a bias that one tends to ignore base rate information (expressed mathematically as prior probability) and focus on specific information (expressed mathematically as 
conditional probability). One tends to focus too much on the information that the result is positive as a result of a test with an accuracy of $99 \%$ (i.e., conditional probability), and misunderstand that such a situation is definitely indicative of a sufferer of this disease without paying attention to the base rate information that roughly $1 \%$ of the population is suffering from this disease (i.e., prior probability). Thus, the posterior probability that Mr. Suzuki is a sufferer of this disease if the test result is positive ( $\operatorname{Pr}($ sufferer|test:+)) tends to be unexpectedly overestimated. Such a bias is called ignorance of base rate.

The participant was required to answer the question below. No time limitation was imposed on the participant. The experimental factor (between-subject factor) was whether debiasing was tried or not. The percentage correct was compared between the biasing and the debiasing condition. The correct answer corresponds to (B).

Probability-based (biasing) representation:

A device has been invented for screening a population for some disease. Although the device is a very excellent one, it is not perfect. Therefore, if someone is not a sufferer, there is a $1 \%$ chance that he or she will be judged positive. Roughly $1 \%$ of the population has the disease. Mr.Suzuki was tested, and the result was positive. Answer the chance (probability) that he is a sufferer.
(A) $85 \%$
(B) $50 \%$
(C) $20 \%$

Frequency-based (debiasing) representation:

Roughly one out of 100 has the disease. A device has been invented for screening a population for the disease. Although the device is a very excellent one, it is not perfect. The device judges that one of 100 is a sufferer in spite of being healthy. How many of 100 are judged positive and how many of these persons is actually a sufferer?

(A) One out of 100 is judged positive, and one is actually a sufferer.

(B) Two out of 100 are judged positive, and one is actually a sufferer.

(C) Five out of 100 are judged positive, and one is actually a sufferer.

\section{Regression to the Mean}

Regression to the mean is explained briefly. Although statistical knowledge teaches us that any extremely good or poor data is likely to regress to the mean over time, we cannot recognize this and tend to illusorily expect that good business or sports achievements at one year continues just the same way the following years. A well-known and famous example of regression to the mean is the Sports Illustrated jinx. The Sports Illustrated is a very popular magazine mostly sold in US. The jinx predicts that an athlete with his or her picture being appeared on the cover of this magazine performs poorly the following year. Some athlete believed in this jinx, and denied to be on the cover when he was required to do so. As such athletes usually achieved excellently better when his or her picture was on the cover, it is a matter of course that the achievement the following year declines, that is, regresses to the mean. The athlete did not recognize regression to the mean, and believed in the jinx. This is a cognitive bias called regression to the mean.

The participant was required to predict the winning average of one NPB (Nippon Professional Baseball Association) pitcher on the basis of data for past two years. The participant was also required to predict the next year's batting average of three NPB players on the basis of data of this year. No time limitation was imposed on 
the participant. The experimental factor (between-subject factor) was whether debiasing was tried or not. The mean difference between the predicted and the actual batting average was calculated and compared between the biasing and the debiasing condition.

Biasing representation:

As shown in Figure 1(a), the prediction of the 3rd year's winning average was conducted with no reference of the mean winning average for past two years. The participant was also ordered to predict next year's batting average of three players A-C on the basis of Table 1(a) with no reference of the mean winning average of three players A-C.

Debiasing representation:

In this condition, as shown in Figure 1(b), the prediction of the 3rd year's winning average was conducted with a reference (horizontal line) of the mean winning average for past two years. The participant was also ordered to predict next year's batting average of three players A-C on the basis of Table 1(b) with a reference of the mean winning average of three players A-C.

(a) Biasing representation

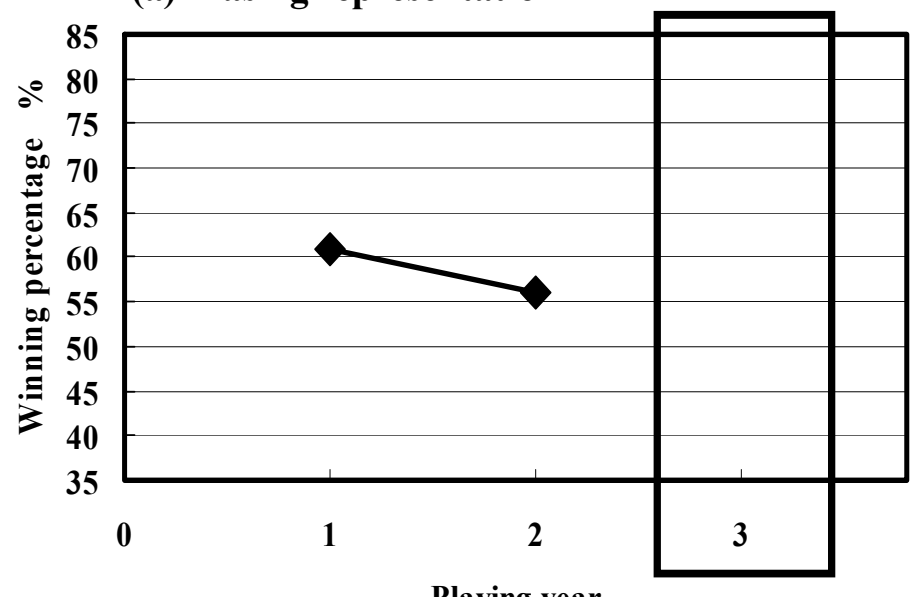

Playing year

(b) Debiasing representation

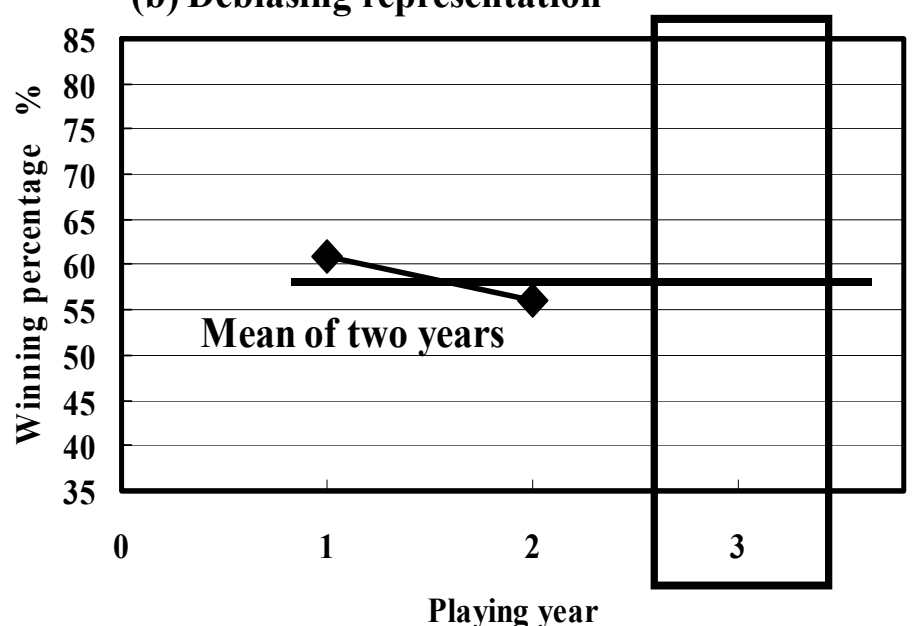

Figure 1. Graphic representation of winning percentage for the past two years: (a) biasing representation, (b) debiasing representation. 
Table 1

Table Representation of Batting Average for Three Players. (a) Biasing Representation, (b) Debiasing Representation

\begin{tabular}{lll}
\hline (a) Biasing representation & & \\
\hline Player & Batting average (this year) & Batting average (next year) \\
\hline A & 0.34 & \\
B & 0.258 & \\
C & 0.239 & \\
\hline (b) Debiasing representation & & Batting average (next year) \\
\hline Player & Batting average (this year) & \\
\hline A & 0.34 & \\
B & 0.258 & \\
C & 0.239 & \\
\hline Mean & 0.279 &
\end{tabular}

\section{Framing Effect}

Framing effect is a phenomenon that a different choice or decision is obtained from the same information depending on how the information is presented to a decision maker. Framing effect is stated briefly using the following Asian disease problem by Kahneman (2011). When the measures are expressed with a positive frame like measures $\mathrm{A}$ and $\mathrm{B}$, it generally tends that a majority of people choose a certain alternative A. Although measures $\mathrm{A}$ and $\mathrm{B}$ are the same as measures $\mathrm{C}$ and $\mathrm{D}$, respectively, we tend to choose measure $\mathrm{D}$ when the measures are presented to us using a negative frame. If we can think rationally and adopt a rational alternative, we should adopt measures $\mathrm{A}$ and $\mathrm{C}$ irrespective of the type of a frame (positive or negative). However, we cannot make a rational choice. In this manner, our choice is different depending on the type of a frame even if the same information is presented to us. This is called framing effect.

The following Asian disease problem was used. The participant was required to read the following sentences and answer to the questions below. Imagine that US is acting against the occurrence of an unusual Asian disease, which is expected to kill 600 people. Two alternative measures have been proposed to combat the disease. It is assumed that the effectiveness (number of people saved) of the measures A and B are estimated as follows when using a positive frame.

Measure A will save 200 people.

Measure B will save 600 people with a probability of one-third.

According to Kahneman (2011), a substantial majority of respondents chose measure A, and preferred the certain option (measure A) to the gamble (measure B). The negative frame can be expressed as follows.

Measure $\mathrm{C}$ will force 400 people into death.

Measure D will save all of 600 people with a probability of one-third, and will force all people into death with a probability of two-thirds.

If we look closely and compare the positive and the negative versions, we easily notice that measures $\mathrm{A}$ and $\mathrm{C}$ are identical, and that measures $\mathrm{B}$ and $\mathrm{D}$ are identical. In spite of this, a large majority of people tended to prefer the gamble (measure D) to measure C. This corresponds to the reversal of preferences, and the framing induces such a cognitive bias. Therefore, it was explored whether such a bias could be deleted or not using the following experimental procedure. No time limitation was imposed on the participant. The experimental factor (between-subject factor) was whether debiasing was tried or not. 
Biasing condition:

The participant allocated to the debiasing condition was provided with the positive and the negative frames above, and required to choose an alternative A or B, or C or D. No other instructions were given to the participant.

Debiasing condition:

The participant was required to select an alternative $\mathrm{A}$ or $\mathrm{B}$, or $\mathrm{C}$ or $\mathrm{D}$, and to state the reason why he or she chose measures $\mathrm{A}$ or $\mathrm{B}$, or $\mathrm{C}$ or $\mathrm{D}$. This will be called elaboration condition.

\section{Illusion of Covariation}

The illusion of covariation (Glymour, 1999) is to inaccurately perceive the relationship between two completely unrelated events. Due to this cognitive bias, we sometimes misunderstand the cause and the effect of some event. From Table 2(a), the simultaneous probability of dizziness and brain tumor is $160 /(160+40)=$ 0.8. A majority of people tend to misunderstand that the dizziness symptom is related with brain tumor by judging from this probability only. However, the simultaneous probability of symptom of no dizziness and brain tumor is also calculated as $40 /(40+10)=0.8$. This definitely indicates that no covariation exists between dizziness and brain tumor. This corresponds to illusion of covariation. From Table 2(b), the simultaneous probability of drinking habit and liver cancer can be calculated as $180 /(180+20)=0.9$. The simultaneous probability of no drinking habit and liver cancer is $30 /(30+170)=0.15$. The simultaneous probability of no drinking habit and no liver cancer is $170 /(30+170)=0.85$. Therefore, through such a scientific approach, it can be concluded that covariation exists between drinking habit and lever cancer. It seems that a majority of people cannot think rationally (scientifically) and get trapped in illusion of covariation.

Table 2

Case (a) Without Causality and (b) with Causality

\begin{tabular}{lcc}
\hline (a) No causality & \multicolumn{2}{c}{ Brain tumor } \\
\cline { 2 - 3 } Dizziness symptom & Yes & No \\
\hline Yes & 160 & 40 \\
No & 40 & 10 \\
\hline (b) Causality & & Liver cancer \\
\hline Drinking habit & & No \\
\cline { 2 - 3 } Yes & Yes & 20 \\
No & 180 & 170 \\
\hline
\end{tabular}

The participant was provided with the data in Tables 2(a)-(b) and required to answer whether there existed causality between dizziness symptom and brain tumor, and between drinking habit and liver cancer. No causality exists in Table 2(a), while there exists causality in Table 2(b). No time limitation was imposed on the participant. The experimental factor (between-subject factor) was whether debiasing was tried or not.

Biasing condition:

The participant was provided with no information necessary for scientifically verifying the causality.

Debiasing condition:

The participant was provided with information (hint) necessary for scientifically verifying the causality. In this condition, the participant was given a brief explanation on the scientific approach to causality. 


\section{Overconfidence}

Overconfidence (Soll \& Klayman, 2004) is to have excessive confidence in one's performance or feasibility of some project or task. This bias is said to be the mother of all types of cognitive biases. Overconfidence is a tendency to be convinced that our judgments and decisions are always correct and hate to be presented with some evidence that our judgments or decisions are wrong. Due to overconfidence, we also tend to think that we are smarter, more attractive, and more competent than we actually are.

Scientific questionnaires which consist of 20 questions were prepared. Examples of scientific questions were as follows.

(1) Which atomic weight is larger, $\mathrm{Ag}$ (silver) or $\mathrm{Au}$ (gold)?

(2) Which condition is $(x-2)(x+3)=0$ for $x=2$, necessary condition or sufficient condition?

(3) Which condition is inequality $x>1$ for $x>-2$, necessary condition or sufficient condition?

(4) Which is larger, 25 or 33 ?

(5) Which wave length is shorter, infrared rays or ultraviolet rays?

(6) Which corresponds to the period of $500 \mathrm{~Hz}, 0.12 \mathrm{~s}$ or $0.002 \mathrm{~s}$ ?

(7) Which probability is higher when drawing a card from the deck of 13 cards from heart 1 to heart 13, (A) the probability of drawing multiples of 5, or (B) the probability of drawing two even numbers in a raw?

(8) How many conditions are there for triangles to be congruent, (A) two, or (B) four?

(9) Which wavelength is smaller, infrared rays or ultraviolet rays?

(10) Which is bigger, 25 or 33 ?

(11) Which is the transformation of frequency of $500 \mathrm{~Hz}, 0.12 \mathrm{~s}$, or $0.002 \mathrm{~s}$ ?

(12) Which is the power when the electrical resistance and the current are $100 \Omega$ and $2 \mathrm{~A}, 400 \mathrm{~W}$ or 800 $\mathrm{W}$ ?

(13) Which is the sequence of prime numbers, (A) 2, 3, 5, 7, 11, 13, ., or (B) $1,2,3,5,7,11, \ldots$ ?

(14) Which of the following is representative of Fleming's left hand rule, (A) voltage, magnetic field, force or (B) voltage, magnetic force, force?

The participant was required to complete 20 questions within 10 minutes. The participant was required to answer one of the following questions after having finished answering all of 20 questions. The experimental factor (between-subject factor) was whether debiasing was tried or not. It was discussed whether overconfidence can be deleted by asking the participant about frequencies of correct answer instead of asking him or her the confidence of the answer.

Biasing condition:

The participant was required to rate his or her confidence to the answer using $0 \%-100 \%$.

Debiasing condition:

The participant was required to predict how many correct answers out of 20 problems he or she reached.

\section{Results}

\section{Conjunction Fallacy}

The comparisons of frequency of conjunction fallacy between biased and the debiased versions are summarized as follows. In the biasing condition, 35 out of 40 participants were got trapped in the conjunction fallacy. On the other hand, in the debiasing condition, only 5 out of 40 participants were got trapped in the conjunction fallacy. A ratio test was used to compare the percentage of got trapped in a cognitive bias of 
conjunction fallacy between the biasing and the debiasing conditions. The percentage correct in the biasing and the debiasing conditions were $12.5 \%$ and $87.5 \%$, respectively. The statistical significance level was set to $1 \%$ or $5 \%$. As a result of a ratio test, the percentage conjunction fallacy was significantly $(z=6.708, p<0.01)$ higher in the biasing condition than in the debiasing condition. It is clear that the debiasing method is effective for deriving correct answers and eliminating conjunction fallacy. The result indicates that the definite distinction between single-event probabilities and frequencies was important for deleting the bias related to conjunction fallacy.

\section{Ignorance of Base Rate}

The frequency-based representation produced more correct answers than the probability-based representation (see Figure 2). A ratio test was used to compare the percentage of got trapped in a cognitive bias of ignorance of base rate between the biasing and the debiasing conditions. The statistical significance level was set to $1 \%$ or $5 \%$. As a result of comparing the percentage correct answer between the probability-based $(40 \%)$ and the frequency-based $(90 \%)$ representation using a ratio test, a significant difference was detected $(z$ $=4.940, p<0.01)$. As hypothesized, the frequency-based representation was effective for eliminating representativeness bias (ignorance of base rate).

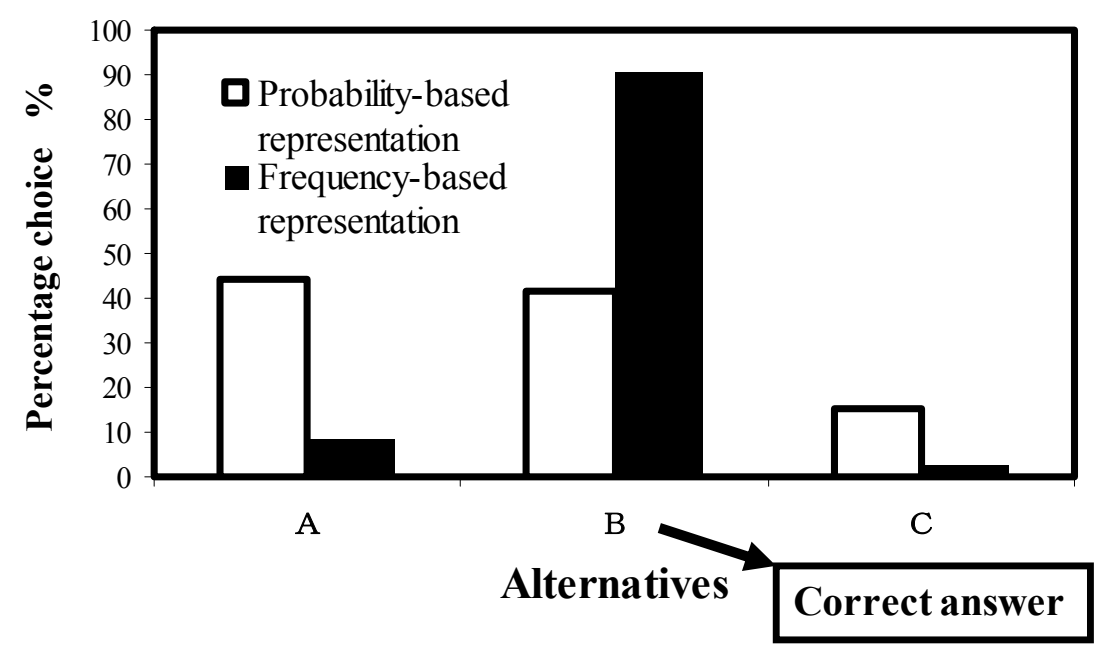

Figure 2. Percentage selection for each alternative (A-C) compared between probability-based and frequency-based representations (ignorance of base rate). In this case, the correct answer is $\mathrm{B}$.

\section{Regression to the Mean}

The difference between the predicted and the actual winning percentage (or batting average) was calculated for each participant. In Figure 3(a), the mean difference between the actual and the predicted value is compared between the biasing (without baseline as shown in Figure 1(a)) and the debiasing (with baseline as shown in Figure 1(b)) condition. In Figure 3(b), the difference between the actual and the predicted value is compared between the biasing (without mean value as shown in Table 1(a)) and the debiasing (with mean value as shown in Table 1(b)) condition. Comparing Figure 3(a) and Figure 3(b), adding a mean value to the figure or the table as a reference was not so effective for eliminating the bias related to regression to the mean. A $t$-test was used to compare the mean difference between the predicted and the actual winning percentage or batting average between the biasing and the debiasing conditions. The statistical significance level was set to $1 \%$ or $5 \%$. As a result of a $t$-test conducted on the difference between the predicted and the actual winning percentage, no 
significant difference was detected between the debiasing (with baseline) and the biasing (without baseline) condition. A similar $t$-test conducted on the difference between the predicted and the actual batting average revealed no significant difference between the debasing (with mean value) and the biasing (without mean value) condition.

(a) Graphic representation

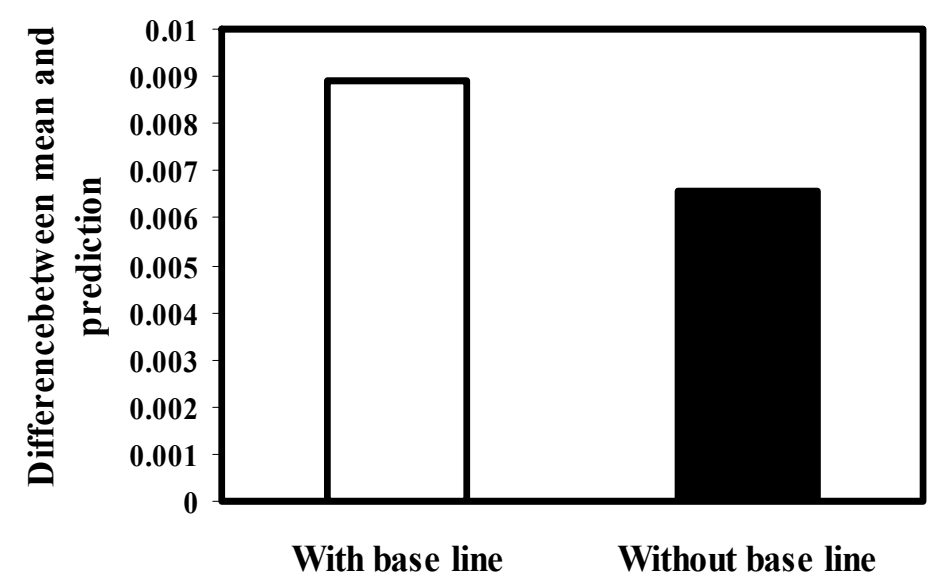

(b) Table representation

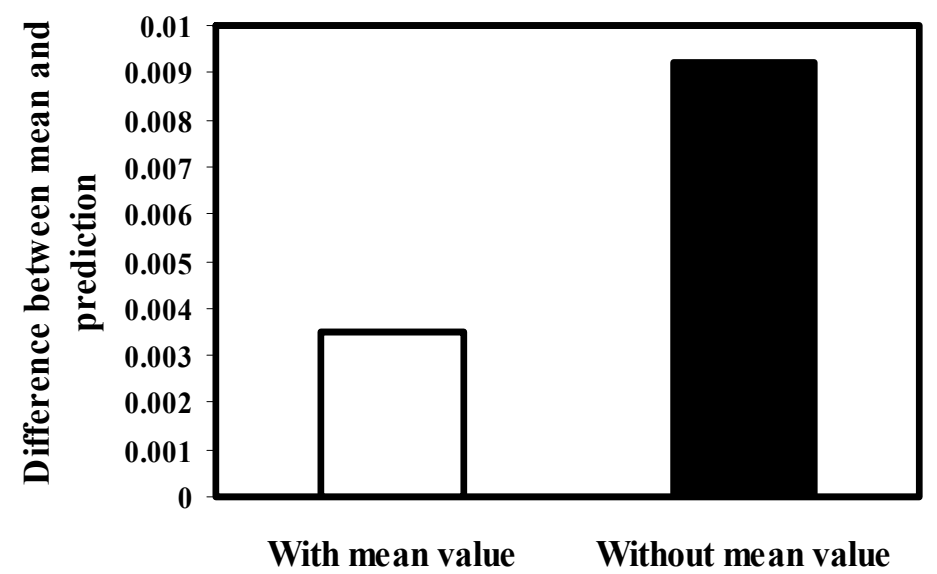

Figure 3. Difference between mean and prediction as a function of debiasing condition (with base line or mean value or without base line or mean value) and representation method ((a) graphic representation and (b) table representation) (regression to the mean).

\section{Framing Effect}

In the elaboration condition (debiasing condition), the participant was required to explain why the alternative was chosen. Under the biasing condition, all participants chose alternative (D) for the negative frame (see Figure 4(a)). As shown in Figure 4(b), this was attenuated to some extent under the debiasing (with elaboration) condition. However, the reversal of preference (Alternative A is selected for positive frame, while alternative $\mathrm{D}$ is selected for negative frame) was not removed by the elaboration instruction to explain why they chose the alternative. 
(a) Without elaboration

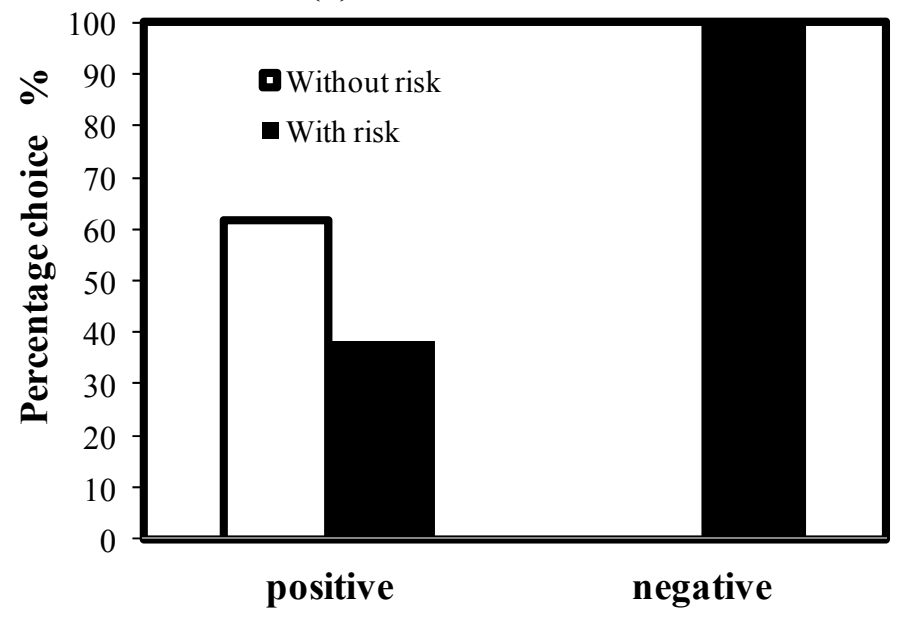

(b) With elaboration

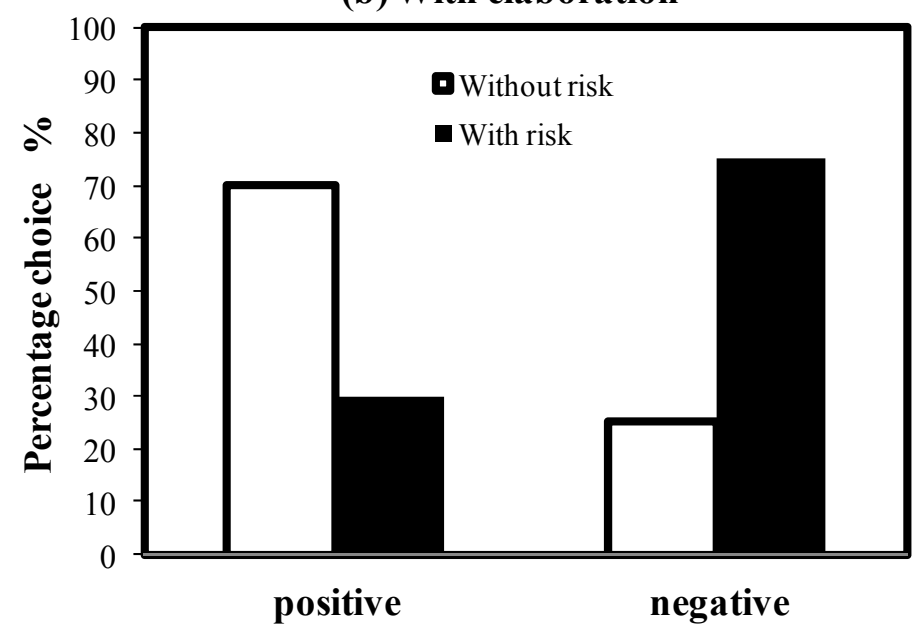

Figure 4. Percentage selection as a function of frame (positive and negative), risk condition (with risk and without risk), and debiasing condition (without elaboration and with elaboration) (Framing effect).

In order to validate this, the following statistical analyses were conducted. For each of the two conditions (biasing and debiasing conditions), the percentage choice was compared between the positive and the negative frame for both sentences with and without risk. A ratio test was used to compare the percentage of got trapped in a cognitive bias of ignorance of base rate between the biasing and the debiasing conditions. The statistical significance level was set to $1 \%$ or $5 \%$.

As for the biasing condition (without elaboration), the percentage choice without risk was $63 \%$ and $0 \%$ for the positive and the negative frame, respectively. The percentage choice with risk was $37 \%$ and $100 \%$ for the positive and the negative frame, respectively. As a result of conducting a ratio test, significant differences were detected between the positive and the negative frame $(z=5.986, p<0.01)$. As for the debiasing condition (with elaboration), the percentage choice without risk was $70 \%$ and $25 \%$ for the positive and the negative frame, respectively. The percentage choice with risk was $30 \%$ and $75 \%$ for the positive and the negative frame, respectively. A similar ratio test revealed a significant difference between the positive and the negative frame $(z$ $=4.029, p<0.01)$. 
As for the biasing condition (without elaboration), the difference of percentage choice between the positive and the negative frame was $63 \%$. The similar difference of percentage choice between the positive and the negative frame was $45 \%$ in case of the debiasing condition (with elaboration). As a result of a ratio test, the difference of percentage choice between the biasing and the debiasing condition was marginally significant $(z=$ $1.615, p=0.053)$. The reversal of preference peculiar to framing effect was not attenuated to a larger extent by the debiasing method.

\section{Illusion of Covariation}

The percentages of correct and wrong answers for both biasing and debiasing conditions are shown in Figure 5(a) and (b). The debiasing measure (providing the participant with elaborated explanation for scientifically verifying the causality) did not work properly for the deletion of cognitive bias in capturing the causality. This shows that it is not so easy for us to recognize the causality correctly, and that we suffer from cognitive biases when understanding the relationship between the cause and effect. As for the (a) no causality condition, the percentages of correct answers for the biasing and the debiasing condition were $45 \%$ and $50 \%$, respectively. A ratio test was used to compare the percentage of got trapped in a cognitive bias of ignorance of illusion of covariation between the biasing and the debiasing conditions. The statistical significance level was set to $1 \%$ or $5 \%$. A ratio test revealed no significant difference. Concerning the (b) causality condition, the percentages of correct answers for the biasing and the debiasing condition were $80 \%$ and $63 \%$, respectively. A ratio test revealed a significant difference between the biasing and the debiasing condition $(z=1.684, p<0.05)$. As shown in Figure 5(b), the debiasing method did not work appropriately for removing a cognitive bias of illusion of covariation.

\section{Overconfidence}

Figure 6 shows the relationship between the actual percentage correct and the prediction of number of correct answers (expressed as predicted percentage correct) as a function of the way how the participant expressed his or her confidence (frequency representation: asking the participant about the frequencies of correct answers out of 20 problems, or probability representation: asking him or her the confidence of the answer). If overconfidence is dominant, the greater part of data exists above the diagonal line in Figure 6 . The following tendencies were observed. For the probability representation condition, the percentage overconfidence and the percentage underconfidence were $21.429 \%$ and $64.286 \%$, respectively. A ratio test was used to compare the percentage of got trapped in a cognitive bias of overconfidence between the biasing and the debiasing conditions. The statistical significance level was set to $1 \%$ or $5 \%$. As a result of ratio test, the percentage of underconfidence was significantly higher than that of overconfidence $(z=2.291, p<0.05)$. For the frequency representation condition, the percentage overconfidence and the percentage underconfidence were $65 \%$ and $30 \%$, respectively. A similar ratio test revealed that the percentage of overconfidence was larger than that of undeconfidence $(z=2.216, p<0.05)$.

Overconfidence was dominant for the frequency representation, while underconfidence was dominant for the probability representation. The result might show that overconfidence is deleted by probability representation. However, it must be noted that not the correct evaluation but the underconfidence appeared as a result of trying to delete overconfidence. 
(a) No causality (relationship between dizziness and brain tumor)

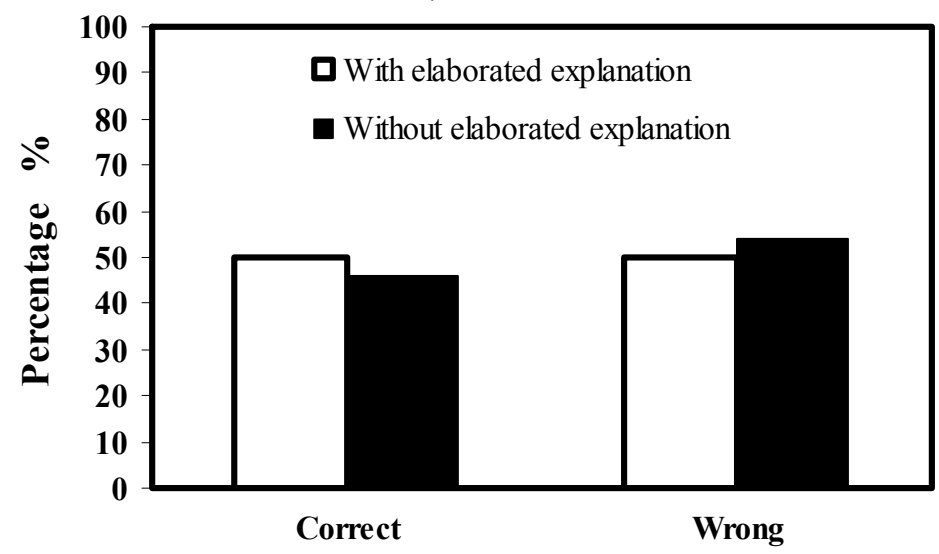

(b) Causality (Relationship between drinking habit and liver cancer)

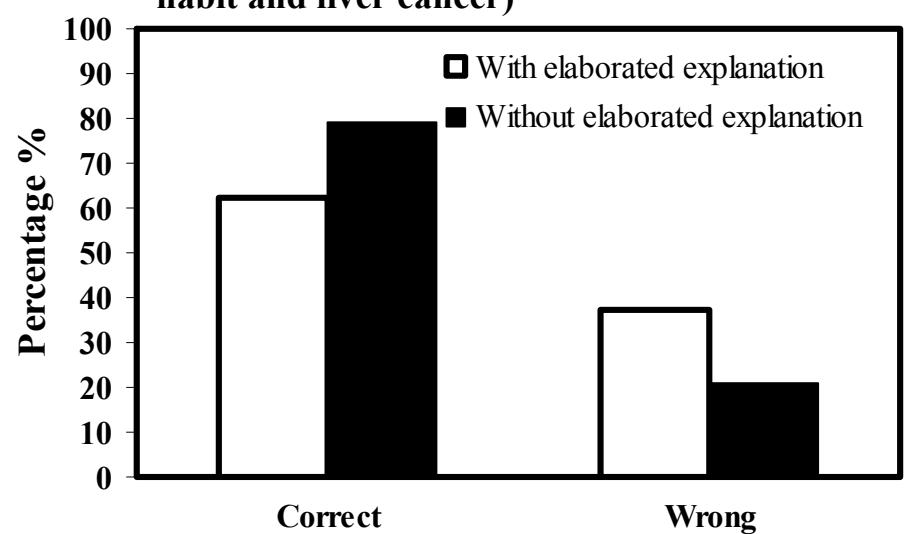

Figure 5. Percentage of correct and wrong answers compared between biasing and debiasing conditions (illusion of covariation). (a) without causality, (b) with causality.

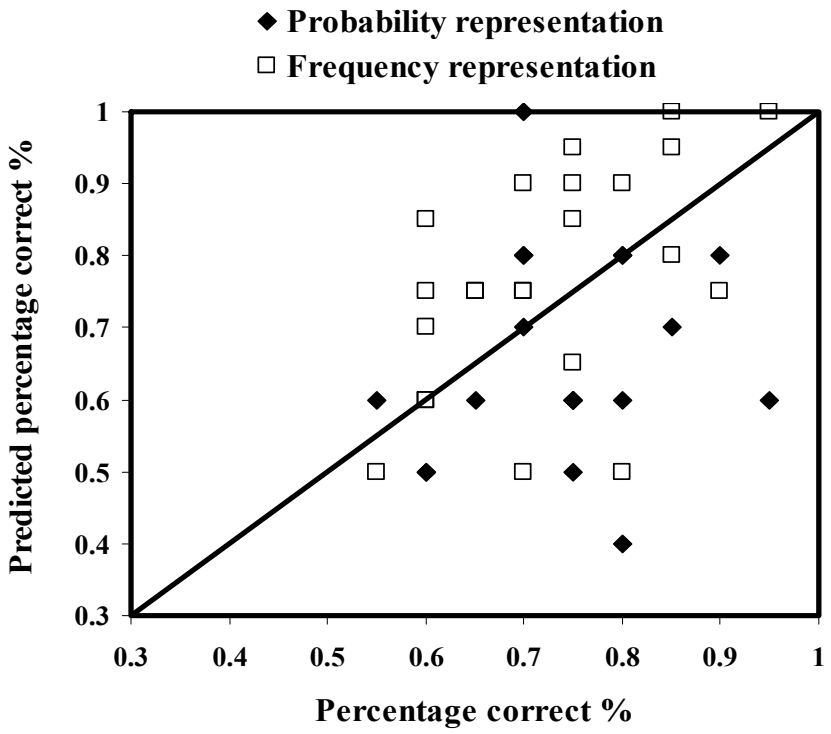

Figure 6. Relationship between the actual percentage correct and the predicted percentage correct. Data above the diagonal line represents overconfidence. Data below the diagonal line represents underconfidence. 


\section{Discussion}

\section{Conjunction Fallacy}

The debiasing method by the frequency-based representation of sentences was effective for deriving a correct answer and eliminating a cognitive bias caused by the probability-based representation. The frequency-based representation can prevent us from being got trapped in a cerebrated reasoning error, that is, misjudgment that the conjunction of two events or facts is larger than the conjunction of one event or fact. As pointed out by Gigrenzer (2007) and Gigerenzer (2015), the frequency-based representation seems to be an effective tool for communicating a genuine risk appropriately.

The finding that the frequency-based expression contributes to delete conjunction fallacy might be effective for deleting cognitive bias especially when evaluating the risks for a variety of events. However, it must be noted that conjunction fallacy was not removed completely by the frequency-representation method.

\section{Ignorance of Base Rate}

As shown in Figure 2, the frequency-based representation was found to be effective for eliminating representativeness bias (ignorance of base rate). Generally, we are not so good at understanding and recognizing probabilistic phenomena, especially risk. In order to overcome this, the frequency-based representation (explanation of probabilistic phenomenon using the frequency-based representation) must be promising. The result indicates that changing the information presentation method from a single-event probability based to a frequency-based one is effective for suppressing and removing the cognitive bias of ignorance of base rate (Gigrenzer, 2007; 2015). The frequency-based representation helped eliminate a cognitive bias of ignorance of base rate due to the probability-based representation.

Taking such characteristics into account, it is desirable to use a frequency-based representation when we need to assess some risk so that cognitive biases are prevented and avoided. This might indicate that as well as conjunction fallacy the frequency-based representation seems to be an effective tool for communicating a genuine risk appropriately. Although the frequency-based representation is effective when choosing from three alternatives (see Figure 2), it must be noted that this does not necessarily assure that we can correctly calculate a probability that Mr. Suzuki is a sufferer on condition that the test result is positive. The following Eq. (1) using Bayes' theorem is necessary to calculate the correct probability. This procedure is a little troublesome. Instead of this formula, the calculation by means of the pie chart in Figure 7 would be more straightforward and be readily understood. Such a graphical representation is compared to expressing the problem based not on a probability but on a frequency. Similarly, conjunction fallacy would be suppressed further by making use of Venn diagram.

$$
\begin{gathered}
P(\text { sufferer } \mid \text { test }:+)=\frac{P(\text { sufferer }) P(\text { test }:+\mid \text { sufferer })}{P(\text { sufferer }) P(\text { test }:+ \text { sufferer })+P(\text { no sufferer }) P(\text { test }:+\mid \text { no sufferer })} \\
=\frac{0.01 * 0.99}{0.01 * 0.99+0.99 * 0.01}=0.5
\end{gathered}
$$




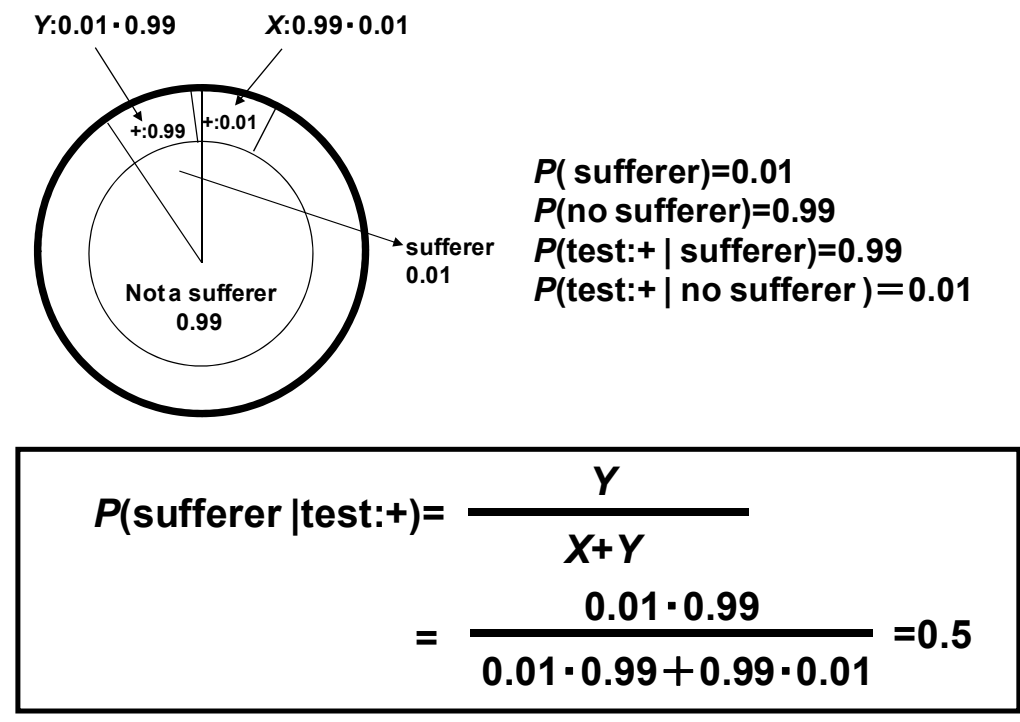

Figure 7. Graphic solution for a question in B. Ignorance of base rate.

\section{Regression to the Mean}

Comparing Figure 3(a) and (b), the table representation seems to be more effective for eliminating representativeness bias (regression to the mean) than the graphic representation. Adding a mean value to the table as a reference seems to be more effective than adding a mean line to the graphic representation for eliminating the bias related to the regression to the mean.

However, it must be noted that the difference between the predicted and the actual batting average revealed no significant difference between the debasing (with mean value) and the biasing (without mean value) condition for both table and graphic representations. This indicates that one cannot conclude that the proposed debiasing method is not necessarily effective like a frequency-based representation for deleting the cognitive bias of conjunction fallacy or ignorance of base rate. The meaning of variance of data and its regression to the mean must be thoroughly understood to prevent us from illusorily imagining growing in values over time such as believing in some stock price continuing to rise. Therefore, the elaborated and scientific explanation or statistical training of the variation and its relevance to the mean might be more effective for eliminating the cognitive bias related to regression to the mean. Future research should verify the effectiveness of such a debiasing technique.

\section{Framing Effect}

The reversal of preference (Alternative A is selected for the positive frame, while alternative $\mathrm{D}$ is selected for the negative frame) was not removed by the elaboration instruction. Although it was shown that a framing effect is a robust property in Kahneman (2011), Tversky and Kahneman (1974), and Kahneman and Tversky (1984), it is not certain whether a framing effect is in fact robust or not. As shown in Figure 4(a) and (b), irrespective of debiasing procedure (elaboration by requiring the respondent (participant) to explain the reason for his or her selection), framing effect (reversal of preference) was not removed so remarkably by requiring the participants to state the reason why they chose the alternatives A or B, or C or D. This might indicate that such an elaboration condition does not work appropriately for removing a framing effect.

Simon, Fagley, and Halleran (2004), on the other hand, insisted that framing effect is not so robust as originally demonstrated by Kahneman (2011), Tversky and Kahneman (1974), and Kahneman and Tversky 
(1984). The elaboration (debiasing) was tried through the explanation why the respondent (participant) chose the alternative. Other elaboration (debiasing) technique such as making the respondent clearly recognize the difference of positive and negative frames might be more effective for deleting such a cognitive bias.

It must be noted that the reversal of preference characteristic of a framing effect was attenuated just a little as shown in Figure 4. At present, it is not certain whether there are more effective methods to eliminate framing effect. The debiasing of framing effect can be summarized as follows. Got Trapped in such a framing effect might lead to the distorted decision making, and thus the judgment of events from a specific frame. This might potentially lead to critical disasters. As mentioned above, merely an elaboration condition did not work appropriately for removing the framing effect. Viewing events from multiple frames will be effective for preventing us from got trapped in a framing effect. It might be possible to eliminate a framing effect completely if we contrive some effective measures to certainly view situations or circumstances from multiple perspectives (frames). The effectiveness of such a method should be validated in future research.

\section{Illusion of Covariation}

It is not so easy for us to recognize the causality (relationship between dizziness and brain tumor, or relationship between drinking habit and liver cancer) correctly. We suffer from cognitive biases when understanding the definite relationship between the cause and effect. When identifying the cause of failures or accidents, such biases are ubiquitous around us. As shown in Figure 5(a) and (b) shows, the attenuation of such a cognitive bias (illusion of covariation) was impossible using the proposed method.

Failing to identify the genuine cause of failures, malfunctions, or accidents frequently induces critical disasters such as the Three Mile Island nuclear power plant accident (nuclear reactor meltdown) (Lusted, 2012; Osif et al., 2006). In the range of our experiment, only a brief explanation for identifying causality did not work effectively at all for deleting fallacy of covariation. This might indicate that it generally takes more time to be able to accurately recognize causal relationship. Continuing to conduct a consistent and scientific training for identifying causality with a high reliability might be effective for deleting such a cognitive bias. Future research should more systematically explore how long it takes to master the accurate identification technique of causality and be free from a cognitive bias of illusion of covariation. As it is very difficult to recognize causality accurately, the results might also suggest that the causality should be carefully judged with the support of experts if there are few people who are accustomed to the identification of causality.

\section{Overconfidence}

The result in Figure 6 might show that overconfidence is deleted by the probability-based representation of confidence of own answer. However, it must be noted that this debiasing method does not lead to correct evaluation of confidence of own answer. In the range of this experiment, underconfidence appeared as a result of deleting overconfidence by means of a debiasing method (probability-based representation). According to Bazerman and Moore (2001), overconfidence leads to illusion of control, fallacy of plan, or optimistic biases. These cognitive biases potentially become a trigger of failures of business such as bankruptcy. Moreover, underconfidence produces passive and negative activities, leads to the insufficiency of a variety of business activation measures, and eventually induces failures of business. The results might indicate that mastering correct knowledge for problem solving by using System 2 that requires us to deliberate is essential for removing over- and under-confidence. Future research should explore how overconfidence or undeconfidence should be deleted. 


\section{General Discussion}

As for conjunction fallacy and ignorance of base rate, the frequency-based representation seems to be an effective tool for communicating a genuine risk appropriately. However, it must be noted that this does not necessarily assure that we can correctly calculate a probability (risk). The pie chart shown in Figure 7 or Venn diagram, which is regarded to be readily understandable as well as a frequency-based representation, must be more effective for deriving a correct value.

The proposed debiasing method by adding to a mean value to a figure or table was not effective for understanding regression to the mean and removing a cognitive bias like a frequency-based representation for deleting the cognitive bias of conjunction fallacy or ignorance of base rate. Further training for understanding the meaning of variance of data and its regression to a mean is necessary to remove a cognitive bias of illusorily imagining growing in values over time (such as believing in achievement of own corporate continuing to rise).

As for the debiasing method of a framing effect, an elaboration condition (merely requiring participants to explain the reason why they chose the alternative) did not work appropriately for removing a framing effect. Rather than such an elaboration method, a systematic training of viewing events or processes from multiple frames (perspectives) might be effective for preventing us from being got trapped in a framing effect. Some effective measures to certainly view situations or circumstances from multiple perspectives (frames) would be effective for eliminating a cognitive bias due to a framing effect. The development of such a measure should be contrived in future research.

The results showed that illusion of covariation cannot be removed easily. Although it is time consuming to master a skill to accurately recognize causal relationship, it is important to continue to conduct a consistent and scientific training for identifying causality with a high reliability for deleting a cognitive bias of illusion of covariation.

The proposed debiasing method did not work effectively for removing overconfidence. Therefore, only steadily mastering correct knowledge for problem solving by using System2 (deliberate and time consuming thinking) might be a necessary condition for removing over- and under-confidence.

The main finding was that cognitive biases cannot be avoided readily. Cognitive biases, in the worst case, distort decision making and lead to a critical disaster or a bankruptcy of a company. When we are forced to make decision mainly using System1, it is impossible for us to avoid cognitive biases. It seems that the judgment or decision by System 1 cannot be released of cognitive biases. Therefore, the judgment or decision by System 1 should be avoided if possible, and the deliberate judgment or decision by System 2 should be practiced. If this is practically impossible, the judgment or decision using System1 should be managed to be less frequent. Future research should further explore whether the cognitive biases could be eliminated to a larger extent, because such biases distort appropriate decision making and induce much damage to organizations or societies as a form of such as a bankruptcy of large companies or critical disasters.

Although it is true that some debiasing methods are effective to some extent, it seems difficult to thoroughly remove cognitive biases ubiquitous in our workplaces or social environment. Future research should develop an effective check sheet for identifying cognitive biases that occur within industrial, social, political, or transportation system and potentially distort our decision so that we can in advance be cautious of cognitive biases or irrational and distorted decision making. 


\section{Cognitive Biases as a Potential Risk Factor of Critical Accidents}

As mentioned in Murata et al. (2015), it is possible that cognitive biases potentially become a trigger of disasters, crashes, collisions, or critical incidences. They, using five case studies, found that the following biases considerably contributed to the distorted decision making, and eventually became main causes of the incident, crash, collision, or disaster: (1) heuristic-based biases, such as confirmation bias, availability bias, regression to mean, groupthink, and social loafing; (2) overconfidence-based biases, such as illusion of control, fallacy of plan, and optimistic bias; and (3) framing effect. Therefore, we must recognize where such biases are ubiquitous within specific man-machine interactions and under what conditions such cognitive biases are likely come into play, distort decision, and in the worst case lead to critical incidents, collisions, crashed, or disasters.

If we succeed in identifying where cognitive biases are likely predominant within specific systems or procedures using a check sheet of cognitive biases, we can introduce some sort of safety interventions or countermeasures that ensure that cognitive biases are removed or do not remarkably manifest themselves as causal factors of critical incidents, crashes, collisions, or disasters. It is therefore necessary to continue to develop debiasing techniques and attempt to remove cognitive biases as risk factors of critical disasters or crashes. By introducing an appropriate safety intervention, these biases can be prevented from becoming causal factors of crashes, collisions, or disasters.

\section{Conclusions}

In the range of this experiment, it seems that some debiasing methods were effective and others were not effective. The conclusions can be summarized as follows.

(1) The debiasing methods of conjunction fallacy and ignorance of base rate using a frequency-based representation were effective for suppressing the biases.

(2) As for framing effect, fallacy of covariation, regression to the mean, and overconfidence, the proposed debiasing methods were not effective.

(3) Although the removal of cognitive biases (debiasing) is important from many perspectives such as the prevention of critical disasters or crashes, or the prevention of a bankruptcy or deterioration of corporation achievements, the debiasing is not readily possible. Therefore, we must continue to develop effective debiasing methods.

Future research should further continue to explore whether such cognitive biases can be deleted or not. Moreover, we must systematically investigate the mechanism how such cognitive biases are linked to the occurrence of undesirable consequences such as the Challenger space shuttle disaster or the Three Mile Island nuclear reactor meltdown.

\section{References}

Altman, M. A. (2012). Behavioral economics for dummies. John Wiley \& Sons Canada, Ltd.

Angner, E. (2012). A course in behavioral economics. Palgrave Macmillan.

Arkes, H. R. (1991). Costs and benefits of judgment errors: Implications for debiasing. Psychological Bulletin, 110, 486-498, 1991.

Bazerman, M. H., \& Moore, D. A. (2001). Judgment in managerial decision making. Harvard University Press.

Gigerenzer, G. (1994). Why the distinction between single-event probabilities and frequencies is important for psychology. In G. Wright \& P. Ayton (Eds.), Subjective probability (pp.129-161), Wiley.

Gigerenzer, G. (2007). Gut feelings—Short cuts to better decision making. Penguin Books.

Gigerenzer, G. (2015). Risk savvy — How to make good decisions. Penguin Books. 
Gigerenzer, G., \& Goldstein, D. G. (1996). Reasoning the fast and frugal way: Models of bounded rationality. Psychological Review, 103, 650-669.

Gigerenzer, G., \& Hoffrage, U. (1995). How to improve Bayesian reasoning without instructions-Frequency formats. Psychological Review, 102, 684-704.

Gigerenzer, G., Todd, P. M., and the ABC Research Group. (1999). Simple heuristics that make us smart. Oxford University Press.

Glymour, C. (1999). Learning causes: Psychological explanations of causal explanation. Minds and Machines, 8, 39-60.

Haselton, M. G., Bryant, G. A., Wilke, A., Frederick, D. A., Gelperin, A., Frankenhuis, W. E., \& Moore, T. (2009). Adaptive rationality: An evolutionary perspective on cognitive bias. Social Cognition, 27, 733-763.

Kahneman, D., \& Tversky, A. (1984). Choices, values, and frames. American Psychologist, 39(4), 341-350.

Kahneman, D. (2011). Thinking, fast and slow. Penguin Books.

Larrick, R. P. (2004). Debiasing. In D. K. Koehler \& N. Harvey (Eds.), Blackwell handbook of judgment \& decision (pp.316-337). Blackwell Publishing.

Lusted, M. A. (2012). The Three Mile Island Nuclear Disaster. Essential Library.

Murata, A., Nakamura, T., \& Kubo, S. (2014). Basic study on prevention of human error-Debiasing method of cognitive biases in decision making. Proc. of AHFE2014, 126-135.

Murata, A., Nakamura, T., \& Karwowski, W. (2015). Influence of cognitive biases in distorting decision making and leading to critical unfavorable incidents. Safety, 1(1), 44-58.

Osif, B. A., Baratta, A. J., \& Conkling, T. W. (2006). TMI 25 Years Later-The Three Mile Island Nuclear Power Plant Accident and its impact. The Pennsylvania State University Press.

Reason, J. (1990). Human error. Cambridge University Press.

Simon, A. F., Fagley, N. S., \& Halleran, J. G. (2004). Decision framing: Moderating effects of individual differences and cognitive processing. Journal of Behavioral Decision Making, 17(2), 77-93.

Soll, J. B., \& Klayman, J. (2004). Overconfidence in interval estimates. Journal of Experimental Psychology: Learning, Memory, and Cognition, 30, 299-314.

Todd, P. M., \& Gigerenzer, G. (2000). Precis of simple heuristics that makes us smart. Behavioral and Brain Sciences, 23, 727-780.

Tversky, A., \& Kahneman, D. (1974). Judgment under uncertainty: Heuristics and biases. Science, 185, 1124-1131.

Tversky, A., \& Kahneman, D. (1983). Extensional versus intuitive reasoning: The conjunction fallacy in probability judgment. Psychological Review, 90, 293-315.

Vaughan, D. (1997). The challenger launch decision: Risky technology, culture, and deviance at NASA. University of Chicago Press. 